\title{
Investigating Hardware Micro-Instruction Folding in a Java Embedded Processor
}

\author{
Flavius Gruian ${ }^{1} \quad$ Mark Westmijze ${ }^{2}$ \\ ${ }^{1}$ Lund University, Sweden \\ flavius.gruian@cs.1th.se \\ ${ }^{2}$ University of Twente, The Netherlands \\ m.westmijze@student.utwente.nl
}

Java Technologies for Real-time and Embedded Systems, 2010 


\section{Outline}

(1) Introduction

(2) Folding BlueJEP

(3) Implementation and Experiments

(4) Discussion

(5) Conclusion 


\section{What are we trying to do?}

Implement bytecode folding on an existing Java embedded processor and evaluate the results with respect to:

- theoretical estimates

- absolute speed-up

- performance w.r.t. device area 


\section{What are we trying to do?}

Implement bytecode folding on an existing Java embedded processor and evaluate the results with respect to:

- theoretical estimates

- absolute speed-up

- performance w.r.t. device area

\section{Finally...}

Is it worth it? 
Starting Point

\section{Original Processor Architecture}

\section{BlueJEP}

BlueSpec System Verilog Java Embedded Processor, a redesign of JOP [M. Schöberl]

- micro-programmed, stack machine core

- predictable rather than high-performance (RT systems)

- JOP micro-instruction set (for ease of programming) 
Starting Point

\section{Original Processor Architecture}

\section{BlueJEP}

BlueSpec System Verilog Java Embedded Processor, a redesign of JOP [M. Schöberl]

- micro-programmed, stack machine core

- predictable rather than high-performance (RT systems)

- JOP micro-instruction set (for ease of programming)

- specified in BSV [see JTRES 2007] 
BlueJEP Architecture

\section{Six Stages Micro-Programmed Pipeline}

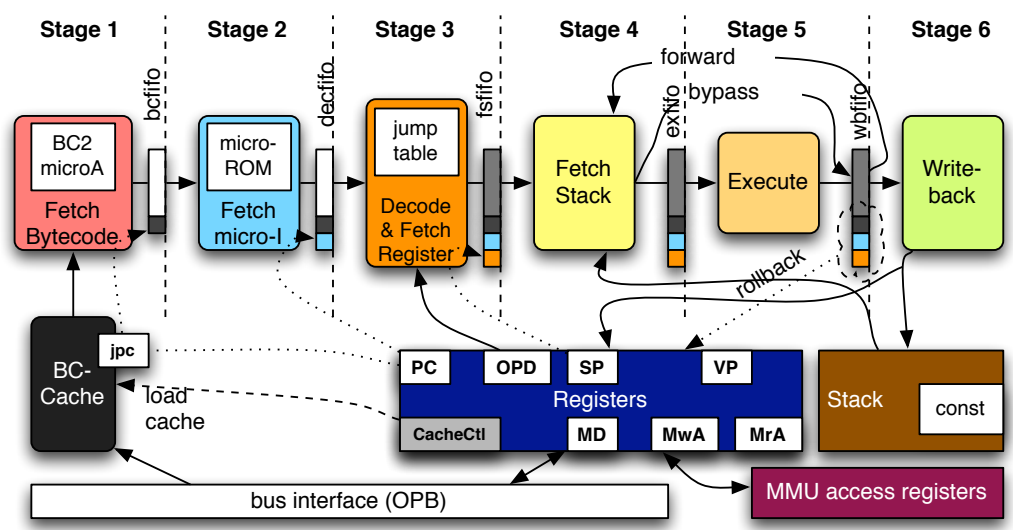


Folding Theory

\section{Bytecode Folding Theory}

- stack machine (JVM) code can be shorter on multi-address machines that emulate them

\begin{tabular}{|c|c|}
\hline $\begin{array}{c}\text { stack code } \\
\approx 7 \text { bytes }\end{array}$ & $\begin{array}{c}3 \text {-address code } \\
\approx 4 \text { bytes }\end{array}$ \\
\hline \hline $\begin{array}{c}\text { iload } \mathrm{a} \\
\text { iload } \mathrm{b} \\
\text { iadd } \\
\text { istore } \mathrm{c}\end{array}$ & add $\mathrm{a}, \mathrm{b}, \mathrm{c}$ \\
\hline
\end{tabular}




\section{Bytecode Folding Theory}

- stack machine (JVM) code can be shorter on multi-address machines that emulate them

\begin{tabular}{|c|c|}
\hline $\begin{array}{l}\text { stack code } \\
\approx 7 \text { bytes }\end{array}$ & $\begin{array}{l}\text { 3-address code } \\
\approx 4 \text { bytes }\end{array}$ \\
\hline $\begin{array}{l}\text { iload a } \\
\text { iload b } \\
\text { iadd } \\
\text { istore } c\end{array}$ & add $a, b, c$ \\
\hline
\end{tabular}

- folding pattern length depends on the available resources (ALUs, memory ports) 


\section{Bytecode Folding Theory}

- stack machine (JVM) code can be shorter on multi-address machines that emulate them

\begin{tabular}{|c|c|}
\hline $\begin{array}{c}\text { stack code } \\
\approx 7 \text { bytes }\end{array}$ & $\begin{array}{c}3 \text {-address code } \\
\approx 4 \text { bytes }\end{array}$ \\
\hline \hline iload $\mathrm{a}$ & add $\mathrm{a}, \mathrm{b}, \mathrm{c}$ \\
iload $\mathrm{b}$ & \\
iadd & \\
istore $\mathrm{c}$ & \\
\hline
\end{tabular}

- folding pattern length depends on the available resources (ALUs, memory ports)

- bytecodes are grouped in classes by resource access, e.g.:

$P$ producer: pushes a value in the stack

$C$ consumer: pops a value in the stack

$\mathrm{O}$ operation: uses top two and pushes back a result

S special: not foldable (breaks a pattern) 


\section{Folding Scheme}

\section{Adopted Folding Scheme}

- fixed folding pattern approach [picoJava-II]

- micro-instruction level (rather than bytecode level)

- maximum length of four micro-instructions (at most four single instruction bytecodes)

\begin{tabular}{|l|r|}
\hline Folding Pattern & Length \\
\hline \hline ppoc & 4 \\
poc & 3 \\
ppc & 3 \\
pc & 2 \\
oc & 2 \\
po & 2 \\
\hline
\end{tabular}


Folding Scheme

\section{Pre-design Estimates}

How much is the number of executed clock cycles reduced? 


\section{Pre-design Estimates}

How much is the number of executed clock cycles reduced? Processed cycle accurate simulation traces say:

- $\approx 30 \%$ fewer cycles for 0-delay memory

- $\approx 25 \%$ fewer cycles for realistic memory 


\section{Architectural Changes}

Increase fetch parallelism to allow folding:

- wider fetch-bytecode stage: up to four bytecodes must be available simultaneously.

- multiple bytecode FIFOs: to feed the next stage with sequences of bytecodes.

- wider fetch-instruction stage: up to four different micro-addresses must be read simultaneously.

- multiple micro-instruction FIFOs: to provide patterns to the decode stage.

- folding schemes in the decode stage: to identify and handle foldable patterns. 


\section{Design}

\section{Configurability}

Highly configurable architecture:

(1) bytecode bandwidth $(1,2,4)$

(2) micro-instruction bandwidth $(1,2,4)$

(3) foldable patterns 


\section{Configurability}

Highly configurable architecture:

(1) bytecode bandwidth $(1,2,4)$

(2) micro-instruction bandwidth $(1,2,4)$

(3) foldable patterns

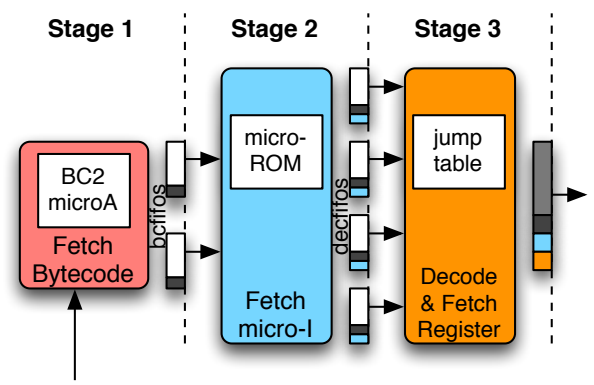

Figure: Handling 2 bytecodes, 4 micro-instructions simultaneously. 


\section{Setup and Tools}

Synthesis $\rightarrow$ device area, maximum clock frequency

- FPGA, Xilinx Virtex-5 (XC5VLX30-3)

- BSV compiler 2006.11, BSV $\rightarrow$ Verilog

- Xilinx EDK 9.1i, Verilog + IPs $\rightarrow$ System

- Xilinx ISE 9.1i, System $\rightarrow$ FPGA

- Chipscope, to calibrate simulation 


\section{Setup and Tools}

Synthesis $\rightarrow$ device area, maximum clock frequency

- FPGA, Xilinx Virtex-5 (XC5VLX30-3)

- BSV compiler 2006.11, BSV $\rightarrow$ Verilog

- Xilinx EDK 9.1i, Verilog + IPs $\rightarrow$ System

- Xilinx ISE 9.1i, System $\rightarrow$ FPGA

- Chipscope, to calibrate simulation

Simulation $\rightarrow$ executed clock cycles

- Desktop, Linux

- BSV compiler 2006.11, BSV $\rightarrow$ Executable

- custom tools for parsing the output from instrumented code 
Results

Original vs. Folding Configurations (2,2; 2,4)

2.5

Relative Clk Cycles

Relative Performance
Relative Clk Frequency

Relative Performance/Area Unit
Relative Device Area

2.0

1.5

1.0
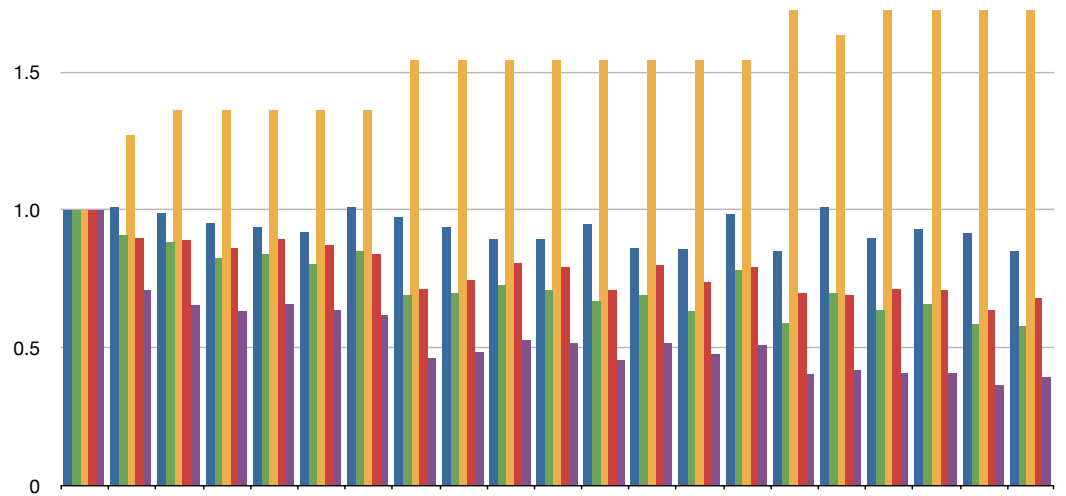
Results

Original vs. Folding Configurations $(4,4)$

2.5

Relative Clk Cycles

Relative Performance

1.5

1.0

0.5

0
Relative Clk Frequency

Relative Performance/Area Unit

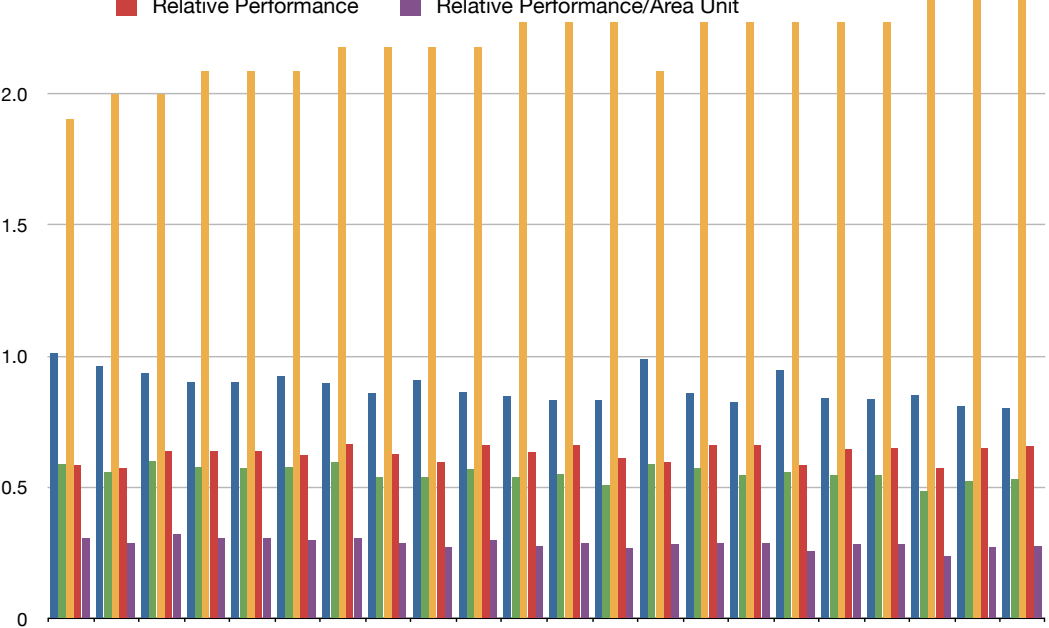




\section{Discussion}

Introducing folding and more patterns:

+ reduce the executed clock cycles (as in theory), but

- ... greatly reduce the maximum clock frequency

- ... and also greatly increase the required device area 


\section{Discussion}

Introducing folding and more patterns:

+ reduce the executed clock cycles (as in theory), but

- ... greatly reduce the maximum clock frequency

- ... and also greatly increase the required device area

Performance/area unit gets as low as $1 / 4$ for some designs with maximal folding!

Introducing more simple processors instead of using folding would be more efficient. 


\section{Provisions}

Reservations:

- using RT-level VHDL instead of BSV may offer better control over the critical path

- introducing more stages may increase clock frequency

- multi-method caches instead of one-method cache would improve overall performance

- other applications than the one we used (GC) could exhibit more folding potential

- more elaborate folding schemes may be more effective 


\section{Finally $\ldots$}

Summary We evaluated folding schemes for BLUEJEP and conclude that the performance greatly decreases although the number of executed cycles is reduced. 


\section{Finally $\ldots$}

Summary We evaluated folding schemes for BLUEJEP and conclude that the performance greatly decreases although the number of executed cycles is reduced.

Observation Theoretical gains are not enough to show efficiency. Complete implementations must be evaluated! 


\section{Finally $\ldots$}

Summary We evaluated folding schemes for BLUEJEP and conclude that the performance greatly decreases although the number of executed cycles is reduced.

Observation Theoretical gains are not enough to show efficiency. Complete implementations must be evaluated!

Recommendation For our case, using several simple processors is potentially more efficient. 
Thank you! 
Thank you!

Questions? 